\title{
Association of a genetic variant of the ZPR1 zinc finger gene with type 2 diabetes mellitus
}

\author{
FUMITAKA TOKORO $^{1}$, REIKO MATSUOKA ${ }^{1}$, SHINTARO ABE $^{1}$, MASAZUMI ARAI ${ }^{1}$, TOSHIYUKI NODA ${ }^{1}$, \\ SACHIRO WATANABE ${ }^{1}$, HIDEKI HORIBE ${ }^{2}$, TETSUO FUJIMAKI ${ }^{3}$, MITSUTOSHI OGURI $^{4}$, \\ KIMIHIKO KATO $^{5}$, SHINYA MINATOGUCHI ${ }^{6}$ and YOSHIJI YAMADA $^{7}$ \\ ${ }^{1}$ Department of Cardiology, Gifu Prefectural General Medical Center, Gifu 500-8717; \\ ${ }^{2}$ Department of Cardiovascular Medicine, Gifu Prefectural Tajimi Hospital, Tajimi, Gifu 507-8522; \\ ${ }^{3}$ Department of Cardiovascular Medicine, Inabe General Hospital, Inabe, Mie 511-0428; ${ }^{4}$ Department of Cardiology, \\ Japanese Red Cross Nagoya First Hospital, Nagoya, Aichi 453-8511; ${ }^{5}$ Department of Internal Medicine, Meitoh Hospital, \\ Nagoya, Aichi 465-0025; ${ }^{6}$ Department of Cardiology, Gifu University Graduate School of Medicine, Gifu 501-1194; \\ ${ }^{7}$ Department of Human Functional Genomics, Life Science Research Center, \\ Mie University, Tsu, Mie 514-8507, Japan
}

Received September 26, 2014; Accepted October 14, 2014

DOI: $10.3892 /$ br.2014.379

\begin{abstract}
Various loci and genes that confer susceptibility to coronary heart disease (CHD) have been identified in Caucasian populations by genome-wide association studies (GWASs). As type 2 diabetes mellitus (DM) is an important risk factor for CHD, we hypothesized that certain polymorphisms may contribute to the genetic susceptibility to CHD through affecting the susceptibility to type $2 \mathrm{DM}$. The purpose of the present study was to examine a possible association of type 2 DM in Japanese individuals with 29 polymorphisms identified as susceptibility loci for CHD by meta-analyses of the GWASs. The study subjects comprised of 3,757 individuals (1,444 subjects with type 2 DM and 2,313 controls). The polymorphism genotypes were determined by the multiplex bead-based Luminex assay, which combines the polymerase chain reaction and sequence-specific oligonucleotide probes with suspension array technology. To compensate for multiple comparisons of genotypes, the criterion of a false discovery rate $($ FDR $) \leq 0.05$ was adopted for testing the statistical significance of the association. The comparisons of allele frequencies by the $\chi^{2}$ test revealed that the rs964184 $(\mathrm{C} \rightarrow \mathrm{G})$ of the ZPR1 zinc finger gene (ZPRI) was significantly associated $(\mathrm{P}=0.0017 ; \mathrm{FDR}=0.050)$ with type $2 \mathrm{DM}$. Multivariable logistic regression analysis with adjustment for age, gender
\end{abstract}

Correspondence to: Professor Yoshiji Yamada, Department of Human Functional Genomics, Life Science Research Center, Mie University, 1577 Kurima-machiya, Tsu, Mie 514-8507, Japan

E-mail: yamada@gene.mie-u.ac.jp

Key words: diabetes mellitus, genetics, polymorphism, ZPR1 zinc finger gene and body mass index revealed that rs964184 of ZPR1 was significantly associated $(\mathrm{P}=0.0012$; odds ratio, 1.25 ; dominant model) with type $2 \mathrm{DM}$ with the minor $G$ allele representing a risk factor for this condition. Fasting plasma glucose levels $(\mathrm{P}=0.0076)$ and blood glycosylated hemoglobin contents $(\mathrm{P}=0.0132)$ significantly differed among ZPRI genotypes with the $G$ allele associated with increases in these parameters. ZPR 1 may thus be a susceptibility locus for type $2 \mathrm{DM}$ in Japanese individuals.

\section{Introduction}

Type 2 diabetes mellitus (DM) is a major public health issue that affects over one billion people worldwide (1). Type 2 DM is a complex disease that involves genetic and environmental factors and their interactions (2). Due to the high prevalence of type 2 DM, identifying the genes or genetic loci associated with the risk or protection of type $2 \mathrm{DM}$ is important for understanding the mechanisms underlying the disease and for benefiting the patients with personalized prevention and treatment programs.

Genome-wide association studies (GWASs) and subsequent meta-analyses have identified $>56$ susceptibility loci for type 2 DM (3-11). However, these susceptibility loci have been identified predominantly in Caucasian populations. Differences in allele frequencies and the effect of size among different ethnicity groups yielded the discovery of new loci in different populations (12). Although several single-nucleotide polymorphisms (SNPs) have been identified as susceptibility loci for type 2 DM in Japanese individuals $(10,11)$, the genes that confer susceptibility to this condition remain to be identified definitively.

Previous GWASs have identified various loci and genes that confer susceptibility to coronary heart disease (CHD) for Caucasian populations $(13,14)$. As type 2 DM is an important risk factor for $\mathrm{CHD}$, we hypothesized that certain 
polymorphisms may contribute to the genetic susceptibility to CHD through affecting the susceptibility to type $2 \mathrm{DM}$. The purpose of the present study was to examine a possible association of type 2 DM in Japanese individuals with 29 SNPs identified as susceptibility loci for CHD by meta-analyses of the GWASs.

\section{Subjects and methods}

Study population. The study population comprised of 3,757 Japanese individuals (1,444 subjects with type 2 DM and 2,313 controls) who either visited outpatient clinics or were admitted to the participating hospitals (Gifu Prefectural General Medical Center, Gifu; Gifu Prefectural Tajimi Hospital, Tajimi; Japanese Red Cross Nagoya First Hospital, Nagoya; Inabe General Hospital, Inabe; Hirosaki University Hospital, Reimeikyo Rehabilitation Hospital and Hirosaki Stroke Center, Hirosaki, Japan) between 2002 and 2012 due to various symptoms or for an annual health checkup. Written informed consent was obtained from all the participants and the Institutional Review Board of each participating hospital approved the study.

Type $2 \mathrm{DM}$ is defined according to the criteria of the World Health Organization, as described previously $(15,16)$. Subjects with type $2 \mathrm{DM}$ had a fasting plasma glucose level of $\geq 6.93 \mathrm{mmol} / 1$ (126 mg/dl), a blood glycosylated hemoglobin (hemoglobin A1c) content of $\geq 6.5 \%$, or were taking anti-diabetic medication. Individuals with type $1 \mathrm{DM}$, maturity onset diabetes of the young, DM associated with mitochondrial diseases or single-gene disorders, pancreatic diseases or other metabolic or endocrinological diseases were excluded from the study. Individuals on medication that may cause secondary DM were also excluded. The control individuals had a fasting plasma glucose level of $<6.05 \mathrm{mmol} / 1(110 \mathrm{mg} / \mathrm{dl})$, a blood hemoglobin A1c content of $<6.2 \%$ and had no history of DM or of receiving anti-diabetic medication.

Selection and genotyping of polymorphisms. SNPs that were recently identified as susceptibility loci for CHD in Caucasian populations were searched for by meta-analyses of GWASs $(13,14)$. These SNPs were examined with the dbSNP database (National Center for Biotechnology Information; http://www.ncbi.nlm.nih.gov/SNP/) to find SNPs with a minor allele frequency of $>0.015$ in a Japanese population. Finally, 29 SNPs (data not shown) were selected and the association with type 2 DM was examined. Wild-type and variant alleles of the SNPs were determined from the original sources.

Venous blood $(7 \mathrm{ml})$ was collected into tubes containing $50 \mathrm{mmol} / 1$ ethylenediaminetetraacetic acid (disodium salt) and genomic DNA was isolated with a kit (Genomix; Talent, Trieste, Italy). Genotypes of the 29 SNPs were determined at G\&G Science (Fukushima, Japan) by a method that combines polymerase chain reaction and sequence-specific oligonucleotide probes with suspension array technology (Luminex Corporation, Austin, TX, USA). The overall call rate of genotyping of 29 SNPs was 99\%. The detailed genotyping methodology was performed as described previously (17).

Statistical analysis. The $\chi^{2}$ test was used to compare the categorical variables, whereas the Mann-Whitney U test was used for analysis of the quantitative data. Allele frequencies of each SNP were compared between subjects with type 2 DM and controls by the $\chi^{2}$ test. A false discovery rate (FDR) was calculated to compensate for multiple comparisons of genotypes, and FDR $\leq 0.05$ was considered to indicate a statistical significance for association. Multivariable logistic regression analysis was performed with type $2 \mathrm{DM}$ as a dependent variable and age, gender ( 0 , women; 1 , men), body mass index (BMI) and the genotype of SNP as independent variables. The SNP was assessed according to dominant (the combined group of heterozygotes and variant homozygotes verses wild-type homozygotes), recessive (variant homozygotes verses the combined group of wild-type homozygotes and heterozygotes) and two additive [additive 1 (heterozygotes verses wild-type homozygotes) and additive 2 (variant homozygotes verses wild-type homozygotes)] genetic models. As fasting plasma glucose level and blood hemoglobin A1c content were not normally distributed $(\mathrm{P}<0.01$ by the Kolmogorov-Smirnov Lilliefors test), these parameters were compared among genotypes by the non-parametric Kruskal-Wallis test. Statistical analysis was performed with JMP version 11 and JMP Genomics version 6.0 software (SAS Institute, Cary, NC, USA).

\section{Results}

Clinical characteristics of the study subjects. The clinical characteristics of the study subjects are shown in Table I. Age, the frequency of males, BMI, the prevalence of smoking, myocardial infarction, dyslipidemia and hypertension, as well as serum concentrations of triglycerides and creatinine, were higher, whereas the serum concentrations of high-density lipoprotein (HDL) cholesterol were lower in subjects with type 2 DM compared to controls.

Associations of SNPs to type 2 DM. Allele frequencies were compared between subjects with type 2 DM and controls by the $\chi^{2}$ test and five SNPs with $\mathrm{P}<0.05$ are shown in Table II. Among these SNPs, rs964184 $(\mathrm{C} \rightarrow \mathrm{G})$ of the ZPR1 zinc finger gene $(Z P R l)$ was significantly $(\mathrm{FDR} \leq 0.05)$ associated with the prevalence of type $2 \mathrm{DM}$. The genotype distributions of five SNPs were in Hardy-Weinberg equilibrium ( $\mathrm{P}>0.05)$ among subjects with type $2 \mathrm{DM}$ and controls.

Multivariable logistic regression analysis with adjustment for age, gender and BMI revealed that rs964184 of ZPRI was significantly associated with type $2 \mathrm{DM}$ in the dominant and additive 1 and 2 models, with the minor $G$ allele representing a risk factor for this condition (Table III). As hypertriglyceridemia is an important risk factor for type $2 \mathrm{DM}$, additional multivariable logistic regression analysis was performed with adjustment for serum triglycerides concentrations or hypertriglyridemia (serum concentration of triglycerides $\geq 1.65 \mathrm{mmol} / \mathrm{l}$ or taking anti-dyslipidemic medication) in addition to age, gender and BMI (Table III). rs964184 was also significantly associated with type $2 \mathrm{DM}$ in the dominant and additive 1 models in this analysis.

Associations of rs964184 to fasting plasma glucose level and blood hemoglobin Alc content. Finally, the associations of rs964184 genotypes to fasting plasma glucose level and blood 
Table I. Characteristics of the subjects with type 2 diabetes mellitus (DM) and controls.

\begin{tabular}{lccr}
\hline Characteristic & Type 2 DM & Controls & P-value \\
\hline No. of subjects & 1444 & 2313 & $<3.1 \pm 11.3$ \\
Age, years & $65.8 \pm 10.1$ & $57.1 / 42.9$ & $<0.0001$ \\
Gender, male/female $(\%)$ & $67.0 / 33.0$ & $23.6 \pm 3.4$ & $<0.0001$ \\
Body mass index, $\mathrm{kg} / \mathrm{m}^{2}$ & $24.1 \pm 3.7$ & 25.5 & 0.0005 \\
Current or former smoker, $\%$ & 30.3 & 36.5 & 0.0013 \\
Myocardial infarction, $\%$ & 66.3 & 42.1 & $<0.0001$ \\
Dyslipidemia, $\%$ & 55.7 & 56.9 & $<0.0001$ \\
Hypertension, $\%$ & 75.5 & $1.43 \pm 0.87$ & $<0.0001$ \\
Serum triglycerides, $\mathrm{mmol} / \mathrm{l}$ & $1.79 \pm 1.39$ & $1.39 \pm 0.43$ & $<0.0001$ \\
Serum HDL-cholesterol, $\mathrm{mmol} / \mathrm{l}$ & $1.25 \pm 0.35$ & $3.10 \pm 0.87$ & $<0.0001$ \\
Serum LDL-cholesterol, $\mathrm{mmol} / \mathrm{l}$ & $3.14 \pm 1.01$ & $84.0 \pm 99.1$ & 0.6136 \\
Serum creatinine, $\mu$ mol $/ \mathrm{l}$ & $93.8 \pm 106.1$ & $4.96 \pm 0.64$ & $<0.0001$ \\
Fasting plasma glucose, $\mathrm{mmol} / \mathrm{l}$ & $10.46 \pm 3.91$ & $6.0 \pm 1.2$ & $<0.0001$ \\
Blood hemoglobin Alc, $\%$ & $7.8 \pm 2.0$ & $<0.0001$
\end{tabular}

Quantitative data are mean \pm standard deviation. HDL, high-density lipoprotein; LDL, low-density lipoprotein.

Table II. Comparison of the single-nucleotide polymorphism $(\mathrm{P}<0.05)$ allele frequencies by the $\chi^{2}$ test between subjects with type 2 diabetes mellitus (DM) and controls.

\begin{tabular}{|c|c|c|c|c|}
\hline Variables & Type 2 DM (\%) & Controls (\%) & P-value (allele) & FDR (allele) \\
\hline rs964184 & & & 0.0017 & 0.050 \\
\hline$C C$ & $728(50.4)$ & $1289(55.7)$ & & \\
\hline$C G$ & 601 (41.6) & 869 (37.6) & & \\
\hline$G G$ & $115(8.0)$ & $155(6.7)$ & & \\
\hline Minor allele frequency & 0.29 & 0.26 & & \\
\hline Hardy-Weinberg P & 0.5585 & 0.6024 & & \\
\hline rs12190287 & & & 0.0101 & 0.119 \\
\hline$C C$ & $472(32.7)$ & $833(36.0)$ & & \\
\hline$C G$ & $696(48.2)$ & $1100(47.6)$ & & \\
\hline$G G$ & $276(19.1)$ & $380(16.4)$ & & \\
\hline Minor allele frequency & 0.43 & 0.40 & & \\
\hline Hardy-Weinberg P & 0.4960 & 0.5996 & & \\
\hline rs11556924 & & & 0.0123 & 0.119 \\
\hline$C C$ & $1394(96.5)$ & $2191(94.7)$ & & \\
\hline$C T$ & $49(3.4)$ & $121(5.2)$ & & \\
\hline$T T$ & $1(0.1)$ & $1(0)$ & & \\
\hline Minor allele frequency & 0.02 & 0.03 & & \\
\hline Hardy-Weinberg P & 0.4044 & 0.6098 & & \\
\hline rs6725887 & & & 0.0249 & 0.179 \\
\hline$T T$ & $1413(98.2)$ & $2280(99.0)$ & & \\
\hline$T C$ & $26(1.8)$ & $22(1.0)$ & & \\
\hline$C C$ & $0(0)$ & $0(0)$ & & \\
\hline Minor allele frequency & 0.009 & 0.005 & & \\
\hline Hardy-Weinberg P-value & 0.7295 & 0.8178 & & \\
\hline rs 2075650 & & & 0.0309 & 0.179 \\
\hline$A A$ & $1042(72.2)$ & $1576(68.3)$ & & \\
\hline$A G$ & 359 (24.9) & $666(28.9)$ & & \\
\hline$G G$ & $42(2.9)$ & $65(2.8)$ & & \\
\hline Minor allele frequency & 0.15 & 0.17 & & \\
\hline Hardy-Weinberg P & 0.1051 & 0.5933 & & \\
\hline
\end{tabular}

FDR, false discovery rate. 
Table III. Multivariable logistic regression analysis of rs964184 of ZPR1 zinc finger gene and type 2 diabetes mellitus with additional adjustments to age, gender and BMI.

\begin{tabular}{|c|c|c|c|c|c|c|c|c|}
\hline \multirow[b]{2}{*}{ Additional adjustments } & \multicolumn{2}{|c|}{ Dominant } & \multicolumn{2}{|c|}{ Recessive } & \multicolumn{2}{|c|}{ Additive 1} & \multicolumn{2}{|c|}{ Additive 2} \\
\hline & P-value & OR $(95 \% \mathrm{CI})$ & $\mathrm{P}$-value & OR $(95 \% \mathrm{CI})$ & $\mathrm{P}$-value & OR $(95 \% \mathrm{CI})$ & P-value & OR $(95 \% \mathrm{CI})$ \\
\hline None & 0.0012 & $1.25(1.09-1.43)$ & 0.1134 & $1.23(0.95-1.59)$ & 0.0036 & $1.23(1.07-1.42)$ & 0.0280 & $1.35(1.03-1.75)$ \\
\hline $\begin{array}{l}\text { Serum concentrations } \\
\text { of triglycerides }\end{array}$ & 0.0209 & $1.18(1.02-1.35)$ & 0.4105 & $1.12(0.86-1.45)$ & 0.0304 & $1.18(1.01-1.35)$ & 0.1923 & $1.20(0.91-1.57)$ \\
\hline Hypertriglyceridemia & 0.0101 & $1.19(1.04-1.37)$ & 0.2677 & $1.16(0.89-1.50)$ & 0.0188 & $1.19(1.03-1.37)$ & 0.1047 & $1.25(0.95-1.64)$ \\
\hline
\end{tabular}

Hypertriglyridemia was defined as a serum concentration of triglycerides $\geq 1.65 \mathrm{mmol} / 1$ or taking anti-dyslipidemic medication. OR, odds ratio; CI, confidence interval; BMI, body mass index.

Table IV. Association of rs964184 of ZPR1 zinc finger gene to fasting plasma glucose level and blood hemoglobin A1c content as determined by the Kruskal-Wallis test.

\begin{tabular}{lcccr}
\hline & \multicolumn{3}{c}{ Genotype of rs964184 } & \\
\cline { 2 - 4 } Parameter & $C C$ & $C G$ & $G G$ & P-value \\
\hline $\begin{array}{l}\text { Fasting plasma } \\
\text { glucose, mmol/1 }\end{array}$ & $6.9 \pm 3.3$ & $7.1 \pm 3.4^{\mathrm{a}}$ & $7.3 \pm 3.8^{\mathrm{a}}$ & 0.0076 \\
$\begin{array}{l}\text { Blood hemoglobin } \\
\text { Alc, } \%\end{array}$ & $6.8 \pm 1.8$ & $6.9 \pm 1.7$ & $7.3 \pm 2.2^{\mathrm{a}}$ & 0.0132 \\
\hline
\end{tabular}

${ }^{a} \mathrm{P}<0.05$ vs. $C C$.

hemoglobin A1c content were examined by the Kruskal-Wallis test (Table IV). rs964184 was significantly associated with the two parameters and the $G$ allele was associated with the increases in fasting plasma glucose level and in blood hemoglobin A1c content.

\section{Discussion}

The associations of 29 SNPs identified as susceptibility loci for CHD by meta-analyses of GWASs to type 2 DM were examined and it was observed that rs964184 of ZPRI was significantly associated with type $2 \mathrm{DM}$ in Japanese individuals. The prevalence of type $2 \mathrm{DM}$, fasting plasma glucose level and blood hemoglobin A1c content were increased by 18.0, 6.7 and 7.4\%, respectively, for individuals with the $G G$ genotype of rs 964184 compared to those with the $C C$ genotype.

rs964184 is located in the intron region of ZPRI at chromosome 11q23.3. ZPR1 is an essential regulatory protein for cell proliferation and signal transduction and may have multiple physiological functions $(18,19)$. The most relevant transcription factor that binds to the promoter region of ZPRI is peroxisome proliferator-activated receptor $\gamma$, which plays an important role in insulin sensitivity and obesity $(20,21)$. The promoter region of ZPRI is also bound by hepatocyte nuclear factor $4 \alpha$, which activates a variety of genes involved in glucose, fatty acid and cholesterol metabolism (22).

$Z P R 1$ is located $\sim 1.6 \mathrm{~kb}$ upstream of the APOA5-A4-C3-AI gene complex. Previous studies have shown that several polymorphisms in or near APOA5 are significantly associated with serum triglycerides concentrations (23-26). rs964184 of ZPRI has been associated with serum triglycerides and this may be attributable to linkage disequilibrium with functional SNPs in APOA5, which influence metabolism of chylomicrons, very-low-density lipoprotein and HDL (27). As an increase in serum triglycerides concentration is an important risk factor for type 2 DM (28), a multivariable logistic regression analysis was performed with adjustment for serum triglycerides levels or hypertriglyceridemia in addition to age, gender and BMI. There was a significant association of rs964184 with type 2 DM in this analysis, indicating that the association was independent, at least in part, of serum triglycerides levels in the study. The previous GWASs suggested that APOA5 polymorphisms may also play an important role in the development of type 2 DM $(29,30)$. A subgroup analysis by ethnicity of a meta-analysis revealed a significant association of the $-1131 \mathrm{~T} \rightarrow \mathrm{C}$ polymorphism of APOA5 with type $2 \mathrm{DM}$ in Asian populations (31). This observation may support the hypothesis that rs964184 of ZPR 1 is associated with type $2 \mathrm{DM}$ through the interaction with APOA5 in Japanese individuals.

Although the contribution of rs964184 to the increased susceptibility to type 2 DM was examined in several GWASs mainly with Caucasians, the significant association was not detected $(32,33)$. The reason for the discrepancy between the previous studies and the present results remains unclear. The variations in the minor $G$ allele frequencies due to the ethnic differences may be, at least in part, responsible for this discrepancy. The frequencies of the $C G$ and $G G$ genotypes of rs9645184 were 23.7 and 2.4\%, respectively, in Caucasian populations (32), whereas in the present study population they were 39.1 and $7.2 \%$, respectively. The $G$ allele of rs964184 was therefore higher in the present population $(26.8 \%)$ compared to the Caucasian population $(13-14 \%)(23,33,34)$. In addition, the prevalence of type $2 \mathrm{DM}$ in the present population was $38.2 \%$, which was more than twice that reported previously (32). The higher frequency of the $G$ allele and the higher prevalence of type $2 \mathrm{DM}$ in the present population compared to those in the previous studies $(33,34)$ may increase the statistical power to detect the association of rs964184 with type $2 \mathrm{DM}$.

In conclusion, the results indicate that rs964184 $(\mathrm{C} \rightarrow \mathrm{G})$ of ZPRI may be a susceptibility locus for type 2 DM in Japanese individuals. Validation of these findings is required in other independent subject panels or ethnic groups. 


\section{Acknowledgements}

The present study was supported by a Collaborative Research Grant from the Gifu Prefectural General Medical Center (no. H24-26 to Y.Y.) and a Grant-in-Aid for Scientific Research from the Ministry of Education, Culture, Sports, Science and Technology of Japan (no. 24590746 to Y.Y.).

\section{References}

1. Unwin N, Gan D and Whiting D: The IDF Diabetes Atlas: proving evidence, raising awareness and promoting action. Diabetes Res Clin Pract 87: 2-3, 2010.

2. Hu FB: Globalization of diabetes: the role of diet, lifestyle, and genes. Diabetes Care 34: 1249-1257, 2011.

3. McCarthy MI: Genomics, type 2 diabetes, and obesity. N Engl J Med 363: 2339-2350, 2010.

4. Shu XO, Long J, Cai Q, et al: Identification of new genetic risk variants for type 2 diabetes. PLoS Genet 6: e1001127, 2010.

5. Kooner JS, Saleheen D, Sim X, et al: Genome-wide association study in individuals of South Asian ancestry identifies six new type 2 diabetes susceptibility loci. Nat Genet 43: 984-989, 2011.

6. Cho YS, Chen $\mathrm{CH}, \mathrm{Hu} \mathrm{C}$, et al: Meta-analysis of genome-wide association studies identifies eight new loci for type 2 diabetes in East Asians. Nat Genet 44: 67-72, 2011.

7. Prokopenko I, McCarthy MI and Lindgren CM: Type 2 diabetes: new genes, new understanding. Trends Genet 24: 613-621, 2008.

8. Tabassum R, Chauhan G, Dwivedi OP, et al: Genome-wide association study for type 2 diabetes in Indians identifies a new susceptibility locus at 2q21. Diabetes 62: 977-986, 2013.

9. Sladek R, Rocheteau G, Rung J, et al: A genome-wide association study identifies novel risk loci for type 2 diabetes. Nature 445 : 881-885, 2007.

10. Yasuda K, Miyake K, Horikawa Y, et al: Variants in KCNQ1 are associated with susceptibility to type 2 diabetes mellitus. Nat Genet 40: 1092-1097, 2008

11. Yamauchi T, Hara K, Maeda S, et al: A genome-wide association study in the Japanese population identifies susceptibility loci for type 2 diabetes at UBE2E2 and C2CD4A-C2CD4B. Nat Genet 42: 864-868, 2010

12. McCarthy MI: The importance of global studies of the genetics of type 2 diabetes. Diabetes Metab J 35: 91-100, 2011.

13. Schunkert H, Konig IR, Kathiresan S, et al: Large-scale association analysis identifies 13 new susceptibility loci for coronary artery disease. Nat Genet 43: 333-338, 2011.

14. CARDIoGRAMplusC4D Consortium; Deloukas P, Kanoni S, et al: Large-scale association analysis identifies new risk loci for coronary artery disease. Nat Genet 45: 25-33, 2013.

15. Expert Committee on the Diagnosis and Classification of Diabetes Mellitus: Report of the expert committee on the diagnosis and classification of diabetes mellitus. Diabetes Care 26 (Suppl 1): S5-S20, 2003.

16. Kuzuya T, Nakagawa S, Satoh J, et al: Report of the Committee on the classification and diagnosis criteria of diabetes mellitus. Diabetes Res Clin Pract 55: 65-85, 2002.

17. Itoh Y, Mizuki N, Shimada T, et al: High-throughput DNA typing of HLA-A, -B, -C, and -DRB1 loci by a PCR-SSOP-Luminex method in the Japanese population. Immunogenetics 57: 717-729, 2005 .
18. Galcheva-Gargova Z, Konstantinov KN, Wu IH, et al: Binding of zinc finger protein ZPR1 to the epidermal growth factor receptor. Science 272: 1797-1802, 1996.

19. Galcheva-Gargova Z, Gangwani L, Konstantinov KN, et al: The cytoplasmic zinc finger protein ZPR 1 accumulates in the nucleolus of proliferating cells. Mol Biol Cell 9: 2963-2971, 1998.

20. Mangelsdorf DJ, Thummel C, Beato M, et al: The nuclear receptor superfamily: the second decade. Cell 83: 835-839, 1995.

21. Corton JC, Anderson SP, Stauber A, et al: Central role of peroxisome proliferator-activated receptors in the actions of peroxisome proliferators. Annu Rev Pharmacol Toxicol 40: 491-518, 2000.

22. Sladek FM, Zhong WM, Lai E, et al: Liver-enriched transcription factor HNF-4 is a novel member of the steroid hormone receptor superfamily. Genes Dev 4: 2353-2365, 1990.

23. Teslovich TM, Musunuru K, Smith AV, et al: Biological, clinical and population relevance of 95 loci for blood lipids. Nature 466: 707-713, 2010.

24. Kathiresan S, Willer CJ, Peloso GM, et al: Common variants at 30 loci contribute to polygenic dyslipidemia. Nat Genet 41: $56-65,2009$.

25. Willer CJ, Sanna S, Jackson AU, et al: Newly identified loci that influence lipid concentrations and risk of coronary artery disease. Nat Genet 40: 161-169, 2008.

26. Waterworth DM, Ricketts SL, Song K, et al: Genetic variants influencing circulating lipid levels and risk of coronary artery disease. Arterioscler Thromb Vasc Biol 30: 2264-2276, 2010.

27. O'Brien PJ, Alborn WE, Sloan JH, et al: The novel apolipoprotein A5 is present in human serum, is associated with VLDL, HDL, and chylomicrons, and circulates at very low concentrations compared with other apolipoproteins. Clin Chem 51: 351-359, 2005.

28. Bernstein RM, Davis BM, Olefsky JM, et al: Hepatic insulin responsiveness in patients with endogenous hypertriglyceridaemia. Diabetologia 14: 249-253, 1978.

29. Talmud PJ, Cooper JA, Hattori H, et al: The apolipoprotein $\mathrm{A}-\mathrm{V}$ genotype and plasma apolipoprotein $\mathrm{A}-\mathrm{V}$ and triglyceride levels: prospective risk of type 2 diabetes. Results from the Northwick Park Heart Study II. Diabetologia 49: 2337-2340, 2006.

30. Soter MO, Gomes KB, Fernandes AP, et al: $-1131 \mathrm{~T}>\mathrm{C}$ and SW19 polymorphisms in APOA5 gene and lipid levels in type 2 diabetic patients. Mol Biol Rep 39: 7541-7548, 2012.

31. Yin YW, Sun QQ, Wang PJ, et al: Genetic polymorphism of apolipoprotein A5 gene and susceptibility to type 2 diabetes mellitus: a meta-analysis of 15,137 subjects. PLoS One 9: e89167, 2014.

32. Kristiansson K, Perola M, Tikkanen E, et al: Genome-wide screen for metabolic syndrome susceptibility loci reveals strong lipid gene contribution but no evidence for common genetic basis for clustering of metabolic syndrome traits. Circ Cardiovasc Genet 5: 242-249, 2012.

33. van de Woestijne AP, van der Graaf Y, de Bakker PI, et al: Rs964184 (APOA5-A4-C3-A1) is related to elevated plasma triglyceride levels, but not to an increased risk for vascular events in patient with clinically manifest vascular disease. PLoS One 9: e101082, 2014.

34. Johansen CT, Wang J, Lanktree MB, et al: Excess of rare variants in genes identified by genome-wide association study of hypertriglyceridemia. Nat Genet 42: 684-687, 2010. 